\title{
Nano-Polymeric Biomaterials Used in Cancer Drug Delivery
}

\author{
Meagan L. Knox ${ }^{\#}$ and Matthew W. Schuppel ${ }^{\#}$ \\ Stephenson School of Biomedical Engineering, University of Oklahoma, Norman OK 73071
}

\begin{abstract}
Cancer drug delivery therapy has become an increasingly researched field. Between the understanding of how nanomedicine can be used in cancer therapies, and the needs of a polymer to deliver drugs to targeted organs and cells, nanopolymers are being used in this field to increase the efficacy of cancer treatments. To use an effective treatment, there must first be an understanding of the inefficiencies of the conventional approaches such as chemotherapy, radiotherapy, and surgery. The properties of an efficient nanopolymer can then be addressed in its degradation properties, bioactivity, bioavailability, biocompatibility, and targeting mechanisms. The use of polymeric micelles, carbon nanotubes, liposomes, dendrimers, etc. have been used in nanomedicine as effective polymers for drug delivery. Modifying these polymers as in PLGA, PEG, and other inorganic polymers has created an environment optimal for loading drugs and incorporating them into cancerous cells. Testing the use of these polymers has decreased the toxicity of the drug therapy and increased the efficiency of nanopolymer treatments. The discussion of the use of polymers in cancer drug delivery and the types of polymers leads to the conclusion that nanopolymers are increasingly being used for cancer therapy and finding significant results in their efficacy. Increasing use of drugs such as paclitaxel loaded into one of the nanopolymers previously mentioned is becoming a method of cancer therapy worth incorporating into practice due to the efficiencies of this cancer treatment.
\end{abstract}




\section{Introduction}

Drug delivery techniques come in many different forms. Through the course of medical development, drug delivery techniques have evolved and changed creating new and improved forms of treating diseases and illnesses such as cancer. In the realm of cancer drug delivery, there has been improvement in the targeting treatments of drug delivery for healing. To develop new and improved delivery techniques, it is important to understand the properties of the materials being used for drug delivery. By manipulating the mechanical properties and understanding the necessities of properties needed in the region of the body being targeted, we can develop new techniques for better approaches. Along with mechanical properties, we can manipulate the degradation properties of the material. In polymers used for drug delivery specifically, we strive to use biodegradable biomaterials so the body can break down the polymer and use the drug as necessary for treatment. With the use of biodegradable materials, we also must focus on the bioinert or bioactive properties of the polymer itself. The activity of the polymer on the body makes a difference in the ability of the drug activity and the desired treatment.

The motivation to understand different nano-polymeric biomaterials used in cancer drug delivery stems from the necessity to create more efficient and effective cancer treatments. Understanding the conventional approaches to cancer treatment, as well as the new approaches, gives physicians a better chance of treating their patients with greater statistical outcomes from the illness. With cancer being one of the most harmful illnesses to humans in recent centuries, it is essential to find new treatments leading to a better chance of survival and remission ${ }^{1}$. Cancer functions by targeting essential organs, which is harmful to the human body causing treatment to be difficult in targeting the correct region without harming the body more $^{2}$. To solve this issue, engineering of nano-polymeric biomaterials has been researched to find an effective way to deliver drugs to regions of interest without harming other regions of the body or healthy cells in that tissue. Many different nano-polymeric biomaterials are used and researched for their different properties and interactions within tissues of the body making this field of large interest to the cancer drug delivery issue. 


\subsection{Biodegradability and Biocompatibility}

When we look into cancer therapies, the common treatments for many years have been the use of radiotherapy, surgery, and chemotherapy. The research into new delivery methods have stemmed from the issues with the inability to dissect all the cancer cells in a surgery, and the toxic side effects of chemo and radiation therapies. Due to the issues that are caused with the conventional approaches to cancer drug therapy, new technologies and medicines have been developed. The use of nanomedicine in creating polymers such as polymeric micelles, carbon nanotubes, liposomes, dendrimers, and more have created a new branch of medicine to treat cancer by using drug delivery in safer and more effective ways. These new polymeric biomaterials used in cancer drug therapy have made treatments more efficient and decreased the number of side effects in cancer patients ${ }^{1}$. When synthesizing polymers, a component of the research is to create a material that is non-toxic. A byproduct of synthesizing a non-toxic polymer for cancer drug delivery is the non-invasive approach of nanoparticles ${ }^{3}$. To create this innovative, non-invasive approach with nanoparticles, we look into their biocompatibility. To achieve biocompatibility, polymers are made from simple monomers naturally present in the body which can then be excreted creating the non-toxicity of the material that is desired for treatments.

Along with creating biocompatible polymers for cancer drug delivery, biodegradability is an important factor. To achieve biocompatibility in the polymer used for drug delivery and provide bioactive molecules to the targeted region of the body, degradation properties are essential to understanding the polymer. A biodegradable material experiences degradation in in vivo applications while continuing to be non-toxic to the patient. To prevent an inflammatory response, the biodegradation can be approached by enzymatic or non-enzymatic pathways. Both natural and synthetic polymers can be created for optimal biocompatibility and biodegradation including, hyaluronan, albumin, polyethylene glycol (PEG), polylactide acid (PLA), and more. Biodegradable nanoparticles are key for drug delivery due to their mechanisms for controlled-release systems (CRS). For non-toxicity and biocompatibility in a biodegradable polymer, it is essential that the properties of the materials cross barriers in the body such as the blood-brain barrier or ophthalmic barrier with molecule concentration control over time. The drug can 
be administered through direct diffusion or from dissociation of the polymer into its monomeric components. The polymer can control the timing of the drug release into its targeted organ or tissue as well as produce the monomeric components, which are the components of degradation, and ultimately be excreted. Different stimuli such as $\mathrm{pH}$, temperature, etc. can have an effect on the degradation of the polymer. Creating $\mathrm{pH}$-switchable polymers have been a novel approach to cancer drug delivery where they can change $\mathrm{pH}$ depending on the region of the body they encounter and ultimately lead to optimal degradation and compatibility in the desired region ${ }^{3}$. In cancer drug therapy, the conventional approaches lead to side effects and toxicity that are not ideal for healing. By using polymeric nanoparticles in cancer drug therapy, we can reduce those effects with different approaches such as encapsulating known cancer drugs with polymers and targeting the cancer cells while considering their degradation and compatibility techniques.

\subsection{Targeting Strategies}

Targeting of cancer cells can be done in two ways, passive targeting and active targeting. Passive targeting uses the release of the drug by using the tumor tissue that passes molecules with either convective or passive diffusion in the cells and interstitial space in the tissue. In most cancers, there is a process called enhanced permeation and retention effect (EPR), which is essentially exploited in the drug delivery processes using cancer drugs. The enhanced permeation and retention effect is a mechanism where nontargeted drugs with a high molecular weight are accumulated in tissues with increased vascular permeability, such as cancer tissues ${ }^{4}$. This then works to release the molecules in the interstitial space due to the permeability of the endothelial lining in the blood vessels from inflammation or hypoxia. Active targeting on the other hand is done through binding nanoparticles to the surface of antibodies, proteins, or peptides in specific locations. The location that is chosen for binding is found from an overexpression of that receptor in the tumor cells ${ }^{3}$. By understanding the cancer cells and the receptors present, passive and active targeting methods can be used for effective localization of the bioactive agent, cancer drug, making the therapy less toxic on healthy tissue, and efficient cancer therapy. Both passive and active targeting are 
shown in figure 1, where we can distinguish between passive diffusion of the drug and active targeting of receptors on the cancer tumors.

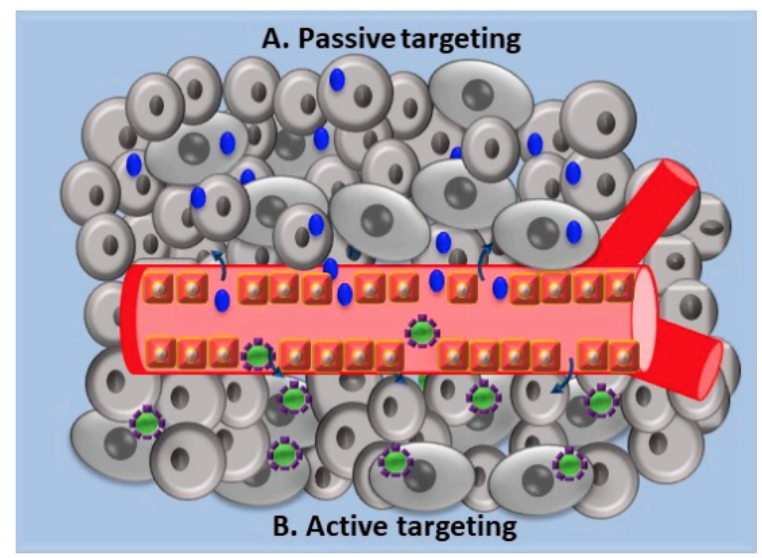

Figure 1.

Shows the difference between passive and active targeting of drugs in cancer therapy with polymeric nanoparticles in cancer tumor tissue . $^{3}$

\section{Background}

\subsection{Conventional Approaches}

The conventional approaches, being chemotherapy and radiotherapy, have been widely used over the last few centuries in cancer treatment. Chemotherapy, Radiotherapy, and surgical removal of cancer tissue is used to target the active cancer cells with a mass removal and the hope of curing the illness. Chemotherapy uses chemicals to kill cells that have a fast proliferation rate; however, it cannot specifically target cancer cells over healthy cells leading to toxicity in the body ${ }^{5}$. The toxins in some chemotherapies can be found in the body to interact with DNA leading to altered protein synthesis which can be mutagenic or carcinogenic potentially causing harm while targeting the cancer ${ }^{6}$. Radiotherapy uses high doses of radiation to kill the cancer cells; however, radiotherapy as well as chemotherapy cannot target the specific cancer cells over healthy cells leading to a diminished immune system and possibly toxicity in the body ${ }^{7}$. Surgical removal of cancer tissue is the removal of the cancerous tissue that is contained to one area of the body. This method is flawed because it is oftentimes unable to remove many areas of cancerous tissue or all of the cancer cells leaving some to proliferate continuing the illness. While these techniques are common and relatively easy to use, they cause significant harm to the body and do not always eliminate the cancer 
cells without depleting essential immune cells needed in the body. This leads us to research and develop an understanding of nano-based cancer treatments for a more effective and efficient treatment targeting cancer cells while leaving healthy immune cells to help attack the illness.

\subsection{Nano-based Approaches}

In nano-based cancer therapy approaches, polymers are one of the main materials used as well as one of the more heavily researched materials. Polymeric materials are preferred because they have potential for surface modifications with chemical transformations, excellent pharmacokinetic control, and provide a good place to trap and deliver therapeutic agents. There are many methods of polymeric drug delivery including, but not limited to, polymeric micelles, nanoparticles, dendrimers, and vesicles. Different methods are used to achieve specific results in terms of loading optimization, biodegradability, bioavailability, and optimal release control.

\subsubsection{Polymeric Materials: Polymeric Micelles}

One of the nanocarriers that is widely used for drug delivery in cancer therapy is polymeric micelles or PMs. PMs are nanocarriers that are formed by spontaneous arrangement of amphiphilic block copolymers in aqueous solutions ${ }^{9}$. Polymeric micelles have been used to carry drugs to tumors. These nanoparticles have a hydrophobic core and a hydrophilic shell where the hydrophobic drugs are loaded into the core to improve the solubility of these drugs. PM carriers provide many advantages including ease of preparation and ability to be customized to fit specific needs for a particular target. To improve drug uptake for specific cells, targeting ligands can be attached to polymeric micelles helping to prevent side effects from off-target cell uptake. One example of a PM that is used currently is Genexol-PM, it is made up of polyethylene glycol (PEG) - polylactide. This PM carries chemotherapeutic paclitaxel to tumors and is accepted for use in the United States.

Polymeric micelles are customizable from the start. Different intermolecular forces that drive the micelle formation can cause the block copolymer micelles to form different categories. There are polyion complex micelles, hydrophobically assembled amphiphilic micelles, and metal complexion rooted micelles. The hydrophobic assembled micelles form the hydrophobic core and hydrophilic shell previously 
mentioned. PMs can also come in a variety of shapes which include rods, vesicles, spheres, and tubules. The shape is dependent on the length of the hydrophilic and hydrophobic block as well as the environment the solvent provides. The morphology of the micelles is shown to have a major impact on the pharmacokinetic properties of micelles. An example of this is that worm-like filomicelles have about ten times longer circulation time in comparison to spherical micelles made of similar material ${ }^{10}$.

Polyethylene glycol (PEG) is the most used hydrophilic part of the micelles for drug delivery purposes. PEG has a molecular weight of 2-15 KDa and is neutrally charged, water soluble, and nontoxic making it an ideal molecule for the body. By using PEG, a hydrophilic protein corona is formed upon the surface of the PMs which helps to remove interactions with unintended components of the blood and improve circulation time of the drug delivery system. This allows more of the drug to reach its intended target. Although PEG is by far the most widely used, there are other molecules, such as polyvinylpyrrolidone (PVP) ${ }^{11}$ and poly(N-isopropylacrylamide) (pNIPAM) ${ }^{12}$, that can be used for the hydrophilic section of PMs.

When constructing the hydrophobic domain of the PMs, biocompatibility and biodegradability are two important factors to consider. Most systems use polymers such as poly(lactic acid) PLA, poly(LLysine) PLL, or poly(beta-amino ester) for this hydrophobic region. To be considered biodegradable, polyesters and polyamides must undergo enzyme catalyzed hydrolysis in vivo.

\subsubsection{Polymeric Materials: Dendrimers}

Dendrimers are another method of polymeric drug delivery used in cancer nano therapy. Dendrimers are branched nanostructures that have an inner core. Both the inner core and outer layer carry the intended drug electrostatically or via covalent bonding. The most common macromolecules used in this area of drug delivery are polyamidoamine (PAMAM), polypropyleneimine, and polyaryl ether ${ }^{13}$. These particles used for dendrimer drug delivery can range in size anywhere between 1 and 100nm but typically tend to be less than $10 \mathrm{~nm}$.

The importance and specialty of dendrimers is based on the heavily branched and globular structure, its defined molecular weight, and ability to control surface functionality all increasing the ability 
for them to transport and successfully deliver drugs. The versatility of dendrimers allows these molecules to deliver both hydrophilic and hydrophobic drugs ${ }^{14}$.

Dendrimers have many advantages including increased solubility and stability, increased half-life, and permeability of drugs. They also can deliver a wide variety of drugs, have targeting abilities, reduced macrophage uptake, and reduced side effects due to more accurate targeting. There are limitations to dendrimers as well. These include inefficient releasing of the desired drug once at location, unstable hydrophobic drug loading, and toxicity due to their size.

Despite the promising research, dendrimer-based cancer therapy drug delivery systems, where a defined dosage is required, is still not available for general medical use. This is mainly due to the lack of ability to synthesize large quantities of clinical grade purity for clinical trials ${ }^{15}$. That being said, there are many ongoing studies and trials that show promising results, and it can be expected for dendrimers as a drug delivery method to be further researched.

\section{Recent Advances}

\subsection{Docetaxel Nanotechnology}

Docetaxel is used in cancer treatments as an antitumor drug. The use of docetaxel with polymeric biomaterials for drug delivery has increased the efficacy of drug delivery in cancer treatments. Combining docetaxel with different nano-polymers has led to the research in nanomedicine on the efficacy of new methods for cancer drug delivery. Docetaxel is an anticancer drug that is widely used in cancer therapy for breast, gastric, ovarian, prostate, and non-benign lung cancers. This drug is used by interacting with cellular microtubule networks key for mitotic and interphase functions, which contributes to great efficacy in antitumor and anti-angiogenic mechanisms. Docetaxel can be combined with each of the following nano polymeric drug carriers to attack cancer and tumor cells within the body and create an overall more effective drug delivery than the conventional approaches to cancer therapies ${ }^{16}$. 


\subsection{Polymeric nanocarriers}

Polymers are found in either organic, inorganic, or microbial fermentation polymer forms such as proteins, peptides, polylactic acid (PLA), poly (lactic-co-glycolic-acid) (PLGA), and polyhydroxybutyrate respectively ${ }^{17}$. Polymers can be modified with liposomes, dendrimers, and micelles for a more effective drug delivery system. A combination of organic and inorganic polymers such as a chitosan-coated PLGA nanoparticle have been used in treatment to deliver proteins to an organ of interest. When PLA nanoparticles are coated in PEG, their stability is increased while exposed to the body, which aids in the transport of the drug to the targeted regions of the body. A type of polymer used in drug delivery is a nanosponge. Nanosponges are created from biocompatible and biodegradable PLGA nanoparticles fused with erythrocyte membrane vesicles. These polymers are made of hyper-cross-linked cyclodextrins, which creates a porous nanoparticle ideal for drug transport. The structure of nanosponges gives them the ability to absorb toxins and other harmful material from tumor cells ${ }^{18}$. The structure and shape along with the negative surface charge of the nanosponge makes them ideal for holding molecules of many sizes for targeting drug resistant cells and increasing effectiveness of chemotherapy treatments ${ }^{19}$. Nanosponges are an ideal cancer drug polymer because they can permeate an erythrocyte membrane to target abnormal cancer cells while decreasing toxicity due to the elimination process through the liver. Another polymer used in cancer drug delivery is an albumin nanoparticle bound to paclitaxel or a derivative of docetaxel. This polymer is commonly used in breast, lung, and pancreatic cancers. The albumin nanoparticle polymer is an effective option for cancer treatments among other polymers because it has increased water solubility and decreasing toxicity. There are many polymer nanoparticles in clinical trials, including the albumin nanoparticle, which are more effective than the conventional approaches by increasing circulation time of the drug and activity within the cancerous tissue. Many different polymers are used in cancer drug delivery for more effective treatments as the solubility, circulation time, biocompatibility, and stability are increased while decreasing toxicity of the treatment itself ${ }^{18}$. 


\subsection{Dendrimers}

One nano polymer that can be used for cancer drug delivery is a dendrimer. A dendrimer is a macromolecule with a core, an interior-branching-dendritic structure, and an exterior surface. The drugs used for treatment can be attached to the dendrimer in its area of compatible solubility for the most effective treatment within the body.

Dendrimers can be used in drug delivery to increase aqueous solubility, pharmacodynamics, and pharmacokinetics. Dendrimers are different from linear polymers in the sense that they are more controllable and have surfaces that can be modified. Passive targeting can be used for drug release in dendrimers with many molecules attached to its surface. Due to the structure, functional groups, and properties of dendrimers, they can be modified to a specific structure as needed for drug delivery. A dendrimer with surface functionalized polyamidoamine (PAMAM) and carboxyl groups immobilize DNA aptamers which targets cancer cells for high efficacy. When PEGylated, a PAMAM dendrimer has also been used in cancer therapy by targeting antitumor activity. Another modified dendrimer used in cancer drug delivery is a methotrexate conjugated PEGylated poly-L-ysine dendrimer, which has been used for treatment in fibrosarcoma tumors. Dendrimers are biocompatible with the body and can be excreted easily which makes them an efficient material to use for drug therapy than the conventional treatments used in cancer treatments as well as comparatively with other nanotechnologies. The PAMAM dendrimer is more effective and biocompatible than a dendrimer on its own because of the amino groups attached to the surface allowing them to circulate longer in the body. Dendrimers can be excreted from the body as readily as molecules such as growth factors and peptides. Although dendrimers are an effective nanotechnology for cancer therapy, they are cytotoxic to normal cells in the body as that is a less desirable property in cancer treatment. However, modifications to dendrimers make the efficacy of them greater than the conventional methods in some cases. Dendrimers can also be used in conjunction with other molecules such as liposomes, nanoparticles, and carbon nanotubes for modified solubility in drug delivery. Each of these 
modifications increases the biocompatibility and efficacy for use with cancer drug delivery and treatments $^{18}$.

\subsection{PLGA}

PLGA (poly-d,l-lactide-co-glycolide) is a widely used biodegradable polymeric material in nanomedicine and nano-cancer therapy because it undergoes hydrolysis in the body to result in lactic acid and glycolic acid. The body can naturally and effectively deal with these two monomers resulting in very little toxicity levels when using PLGA for drug delivery.

Acetone dissolved polymer is added drop by drop to an aqueous phase causing the organic phase to evaporate under reduced pressure. There are many different uses for PLGA nanoparticles including development of: nanovaccines, gene delivery system, nano-antagin, growth factor, and more. Many factors affect the release and effectiveness of the nanomedicines made from PLGA including: surface modification, particle size, drug entrapment methods, and molecular weight. Due to the acidic nature of PLGA, it is not used by itself for drugs or bioactive molecules ${ }^{21}$. There are, however, PLGA nanoparticle blends that allow for them to be used for these purposes including blending it with pectin, chitosan, and alginate.

PLGA has been approved for human use, nanomedicine, and nanotherapy by the USFDA ${ }^{22}$. PLGA is widely used for entrapment of anticancer drugs in cancer nanotherapy. The surface of a PLGA nanoparticle can be modified to target specific tumors or tissues in the body. PLGA nanoparticles of a larger size are best used for multifunctional imaging and probes that include entrapped cancer drugs, release, and targeting in a single nanoparticle system ${ }^{23}$. Although, the efficiency of these nanoparticles are not perfect and other methods are used to improve the performance. The properties of the nanoparticles (shape, size, surface morphology, etc) can be altered to increase the efficiency.

To increase the delivery efficiency of cancer related drugs, PLGA nanoparticles are used to encapsulate them. 9-Nitrocamptothecin (9-NC) is promising in the field of anticancer drugs and provides a unique targeting mechanism for the nuclear enzyme topoisomerase-I. Although, the delivery of 9-NC is difficult due to its low water solubility and instability at biological pH. PLGA was used to entrap 9-NC by nanoprecipitation and this has led to a more than 30\% encapsulation efficiency without the loss of 9-MCs 
anticancer properties ${ }^{24}$. The delivery efficiency in vivo has also led to a more controlled release of 9-MC. Paclitaxel is a drug that causes cell death by disrupting cell division. It is effective against breast, colon, ovarian, and carcinoma cancers. Due to its low solubility, it is less effective for cancer treatment even though it is a potent anticancer agent. PLGA with vitamin E TPGS has been used to encapsulate paclitaxel by solvent evaporation and extraction methods for controlled release. This combination has shown a decent amount of activity and much faster administration than other delivery methods ${ }^{25}$. The release shows a $50 \%$ burst release in the first 24 hours of administration with a slow release for one month in vivo. It is shown that encapsulating paclitaxel in PLGA-TPGS nanoparticles strongly increases delivery efficiency and blood circulation time. An even more improved method of PLGA-TPGS drug delivery system is discussed in the Polymeric Micelles section. Cisplatin is another anticancer drug that crosslinks DNA molecules in ways that interfere with cell division by mitosis. It is a very potent anticancer agent but has limited clinical use due to its toxicity in health issues. Targeted delivery of cisplatin to only cancerous cells would help reduce toxicity levels and improve drug efficiency. Encapsulating cisplatin in PLGA nanoparticles by double emulsion methods have shown increased drug resistance in the blood via intravenous administration ${ }^{26}$. In addition, at the targeted site of the PLGA-mPEG nanoparticles loaded with cisplatin, there has been fast degradation and controlled release of the cisplatin preventing tumor growth.

\subsection{Polymeric Micelles}

Pluronics ${ }^{\circledR}$, otherwise known as poloxamers, are a nonionic block copolymer which has a structure resembling an A-B-A set up. They are made up of hydrophobic propylene oxide (PO) fragments, as well as hydrophilic ethylene oxide (EO) branches. These poloxamers have a central poly(propylene oxide) (PPO) block which forms the hydrophobic core in the micelle where the drug is carried. This core is encompassed on both sides by two hydrophilic chains of poly(ethylene oxides) (PEO) which forms the hydrophilic corona shell of the micelle. Ultimately, this structure looks like PEO-PPO-PEO which is shown below.

Poloxamers have also been used as a protective coating for nanocarriers to improve circulation time in the blood for more effective targeting. The central PPO block is the point that is anchored to the surface of the nanocarrier via hydrophobic interactions. The pluronic class of polymers, or poloxamers, is approved 
by the FDA as an inactive ingredient, a substance added that has no pharmacological effect ${ }^{28}$. Thus, poloxamer has been recognized as a polymer that can be used for an effective drug delivery method. The downside to pluronics is that they can be unstable and form aggregates. That being said, poloxamers have the sought after therapeutic property of multiple drug resistance, which is widely used in nano-polymer anticancer therapy. In light of this, poloxamers have been largely used in the formation of polymeric micelles to deliver chemotherapeutic drugs in cancer therapy.

Zhao et al. has shown the loading of circumin (Cur), an anticancer agent derived from the Curcuma longa plant, in micelles composed of Pluronic P123 and F68 ${ }^{29}$. They saw a highly effective drug entrapment and loading rates of $86.93 \%$ and $6.99 \%$ respectively. They concluded that micelles mixed Pluronic P123 and F68 serve as a highly potential nanocarrier to improve solubility and biological activity of circumin. In another study by Park et al., singlet-oxygen producible polymeric micelles made with Pluronic F127 conjugated with chlorin e6 were produced ${ }^{30}$. The PMs were loaded with Doxorubicin, a cytotoxic chemotherapy medication, as well to further enhance the anticancer properties of chlorin e6. Resistance to Doxorubicin was observed to have lowered without any side-effects thus improving the efficiency of the therapy. In another recent study, a micelle-like structure made of poloxamer-methotrexate (MTX) conjugate has been developed by Ren et al. as a nanocarrier for methotrexate delivery ${ }^{31}$. MTX was entrapped to the micelle-like structure drug delivery system as well as chemically conjugated. Poloxamer-methotrexate (pMTX) was synthesized by MTX conjugating to poloxamer with an ester bond. The hydrophobic nature of the MTX causes it to form the inner core of the p-MTX micelle. The inner core can encapsulate free MTX for delivery. The study found that the micelle-like structure causes the MTX to remain in blood circulation longer as well as prolonged in vivo resistance time compared to solely free MTX. Poloxamer (427) has also been combined with vitamin E TPGS to form micelles for cancer drug delivery systems. These mix micelles carry doxorubicin in the core and the surface is covered with folic acid. This is done by introducing folic acid conjugated to P407 into the micelles. Introducing TPGS into cancer nanotherapy helps reduce multiple drug resistances, provides anticancer properties, and induces apoptosis. Poloxamers have also been shown to reverse many drug resistances; another study incorporated PLGA-TPGS/poloxamer 235 in order to 
overcome many drug resistances in docetaxel resistant human breast cell cancer line ${ }^{32}$. The coating of the poloxamer on the PLGA-TPGS nanoparticles increased uptake of these nanoparticles in otherwise docetaxel resistant MCF-7 human breast cancer cell lines compared to the coatingless PLGA-TPGS nanoparticles. Adding the coating caused the nanoparticles to produce a much higher cytotoxicity of loaded docetaxel, both in vivo and in vitro, than the coatingless PLGA-TPGA nanoparticles including the clinically used docetaxel formulation, Taxotere ${ }^{\circledR}$.

\subsection{Oligomer nanocarriers}

Chitosan and cyclodextrin are polymeric oligomer nanocarriers that have been studied for the use of cancer drug delivery. The properties of both of these molecules contribute to their biocompatibility and efficacy in drug delivery. Chitosan is a bioactive compound, which can be modified for greater solubility in the body and distribution of a desired drug. Due to the solubility of chitosan in acidic $\mathrm{pH}$ environments it can degrade and distribute the drug to tumor cells. The use of chitosan with a cancer drug has less cytotoxicity than other forms of polymeric carriers such as dendrimers, which makes it a favorable material to use in cancer treatments. A modified glycol chitosan nanoparticle loaded with docetaxel, a common cancer drug, was tested in treatment and found to have high drug loading and drug release efficiency. Cyclodextrin is also specifically used in cancer drug delivery due to its ability to interact with an anticancer drug and distribute said drug within the body. Cyclodextrin has a hydrophobic cavity and hydrophilic surface which aids in the ability to hold the drug and interact with the body to release the drug when necessary. The use of cyclodextrin in a nanocarrier increases the solubility, stability, and bioavailability of

the drug into the body within the drug carrier ${ }^{16}$. These oligomer nanocarriers are a new and efficient way of distributing cancer drugs with the use of organic polymers in conjunction with inorganic polymers to increase efficiency of drug delivery to tumor or cancer cells. 


\subsection{Linear Cyclodextrin Polymer}

A certain type of cyclodextrin used in cancer drug delivery is the linear cyclodextrin polymer. As described above, cyclodextrin is an effective compound for drug delivery due to its structure and ability to interact with hydrophobic and hydrophilic environments. In this experiment, a linear cyclodextrin polymer is made by creating a linear high molecular weight cyclodextrin polymer. This structure is made to increase pharmacokinetics, bioavailability, and ultimately increase efficacy of the drug loaded. The cyclodextrin is made into this linear form by a ketal linkage within the molecule, which increases the drug loading efficiency and degradation of the polymer. The ease of degradation of the polymer leads to a decreased toxicity which is more effective in drug treatment ${ }^{33}$.

They have shown increased bioavailability and a decreased elimination from the body which increases the efficacy of the polymer in drug treatment by increasing circulation time of the drug. The polymer also showed an increase in cellular permeability, which leads to a greater permeation of the drug into the targeted region of the body for increased healing thus a lower dose of drug is needed. The polymer also showed a decreased toxicity in the body where negative effects on body weight were limited, which also increases the efficacy of the polymer for use in drug delivery ${ }^{33}$.

\section{Conclusion}

Polymeric biomaterials used for drug delivery in cancer therapy have evolved significantly in the past couple decades. Being a relatively new field, nanotherapeutics lack many FDA approved polymeric solutions. Despite liposomes taking the majority of FDA approved nanotherapeutics, polymeric solutions are heavily researched due to their ability to be customized to fit anticancer drugs as well as customized to improved circulation time in the blood stream leading to more efficient targeting. As described in this paper, there are many polymeric nanomaterials used in drug delivery with promising futures such as micelles, oligomers, PLGA molecules, dendrimers, and other nanocarriers. These polymeric materials help increase bioavailability, permeability, and solubility of many potent anticancer drugs that are otherwise difficult to deliver orally. 
Limitations to the use of nano-polymeric biomaterials in cancer drug delivery include, the inability to image and see nanoparticles and the inability to control the release of the drug molecule.

The inability to image nanoparticles poses a problem when visualizing the distribution of the polymer in tissues and organs. Not all of the organ or tissue can be seen, therefore estimations of the amount of drug being released to the cancerous tissue may be taken and vary the actual effectiveness of the polymer in drug delivery ${ }^{34}$. For the drug to achieve its maximum therapeutic potential, it must be released at the appropriate site. The ability to release the drug when it reaches the cancerous cells makes for less dosages and a lower dosage amount saving the patient from unnecessary exposure to cytotoxic drugs. There is a lack of knowledge in new ways to modify and change polymers to create more effective ways to deliver cancer drugs for treatments. Future work in this field can consist of studying surface modifications to polymers to improve the targeting, circulation time, and release of the anticancer drug. Overall, more research will continue to be done in the field of nanomedicine to create biocompatible polymers with optimal properties for the most effective cancer drug therapies.

\section{Authorship}

\# Meagan L. Knox and Matthew W. Schuppel contributed equally

\section{References}

1. Zhou, Q., Zhang, L., \& Wu, H. Nanomaterials for cancer therapies. Nanotechnology Reviews. 2017. 6(5), 473-496. https://www.degruyter.com/view/journals/ntrev/6/5/article-p473.xml (accessed Oct 29, 2020).

2. Questions People Ask About Cancer. American Cancer Society. 2020. https://www.cancer.org/cancer/cancer-basics/questions-people-ask-about-cancer.html (accessed Oct 29, 2020).

3. Calzoni, E., Cesaretti, A., Polchi, A., Di Michele, A., Tancini, B., \& Emiliani, C. Biocompatible polymer nanoparticles for drug delivery applications in cancer and neurodegenerative disorder therapies. Journal of functional biomaterials. 2019. 10(1), 4 (accessed Oct 29, 2020).

4. Maheshwari N., Tekade R. Guiding Factors and Surface Modification Strategies for Biomaterials in Pharmaceutical Product Development. Biomaterials and Bionanotechnology. 2019. https://www.sciencedirect.com/topics/pharmacology-toxicology-and-pharmaceuticalscience/enhanced-permeability-and-retention-effect (accessed Oct 29, 2020). 
5. Chemotherapy. Mayo Clinic. 2019. https://www.mayoclinic.org/testsprocedures/chemotherapy/about/pac-20385033 (accessed Oct 29, 2020).

6. Moreno, S. N., \& Docampo, R. Mechanism of toxicity of nitro compounds used in the chemotherapy of trichomoniasis. Environmental Health Perspectives. 1985. 64, 199-208. https://ehp.niehs.nih.gov/doi/abs/10.1289/ehp.8564199 (accessed Oct 29, 2020).

7. Radiation Therapy for Cancer. National Cancer Institute. 2018. https://www.cancer.gov/aboutcancer/treatment/types/radiation-therapy (accessed Oct 29, 2020).

8. Venditti, I. Morphologies and functionalities of polymeric nanocarriers as chemical tools for drug delivery: A review. Journal of King Saud University-Science. 2019. 398-411. https://www.sciencedirect.com/science/article/pii/S101836471730770X (accessed Oct 29, 2020).

9. Sosnik, A. CHAPTER 5. Temperature- and PH-Sensitive Polymeric Micelles for Drug Encapsulation, Release and Targeting. Smart Materials Series Smart Materials for Drug Delivery. 2017, 115-147. https://pubs.rsc.org/de/content/chapter/bk978184973877400115/978-1-84973-877-4 (accessed Oct 29, 2020).

10. Geng Y, Dalhaimer P, Cai S, Tsai R, Tewari M, Minko T. Shape effects of filaments versus spherical particles in flow and drug delivery. Nat Nanotechnol. 2007. 249-255. https://www.nature.com/articles/nnano.2007.70 (accessed Oct 29, 2020).

11. Benahmed A, Ranger M, Leroux J. Novel polymeric micelles based on the amphiphilic diblock copolymer poly(N-vinyl-2-pyrrolidone)-block-poly(D,L-lactide). Pharm Res. 2001. 323328. https://link.springer.com/article/10.1023/A:1011054930439 (accessed Oct 29, 2020).

12. Chung J, Yokoyama M, Aoyagi T, Sakurai Y, Okano T. Effect of molecular architecture of hydrophobically modified poly(N-isopropylacrylamide) on the formation of thermoresponsive core-shell micellar drug carriers. J Control Release. 1998. 119-130. https://pubmed.ncbi.nlm.nih.gov/9741919/ (accessed Oct 29, 2020).

13. Frechet J., Tomalia D. Dendrimers and other dendritic polymers. Wiley. 2001. https://www.wiley.com/en-us/Dendrimers and Other Dendritic Polymers-p9780471638506 (accessed Oct 29, 2020).

14. Wolinsky J., Grinstaff M. Therapeutic and diagnostic applications of dendrimers for cancer treatment. Advanced drug delivery reviews, 2008. 1037-1055. https://pubmed.ncbi.nlm.nih.gov/18448187/ (accessed Oct 29, 2020).

15. Yeo Y. Nanoparticulate drug delivery systems: strategies, technologies, and applications. John Wiley and Sons. 2013. https://www.wiley.com/en-us/Nanoparticulate Drug Delivery Systems: Strategies, Technologies, and Applications-p-9781118148877 (accessed Oct 29, 2020).

16. Zhao P, Astruc D. Docetaxel nanotechnology in anticancer therapy. ChemMedChem. 2012. 7(6), 952-972. $\quad$ https://chemistryeurope.onlinelibrary.wiley.com/doi/full/10.1002/cmdc.201200052. (accessed Nov 16, 2020).

17. Yu L, Dean K, Li L. Polymer blends and composites from renewable resources. Prog Polym Sci. 2006. 31:576-602. https://www.sciencedirect.com/science/article/pii/S0079670006000414. (accessed Nov 16, 2020). 
18. Li Z, Tan S, Li S, Shen Q, Wang K. Cancer drug delivery in the nano era: An overview and $\begin{array}{llll}\text { perspectives. Oncology } & \text { reports. }\end{array}$ https://doi.org/10.3892/or.2017.5718 (accessed Nov 16, 2020).

19. Trotta F, Dianzani C, Caldera F, Mognetti B, Cavalli R. The application of nanosponges to cancer drug delivery. Expert Opin Drug Deliv. 2014. 11(6):931-41. https://pubmed.ncbi.nlm.nih.gov/24811423/. (accessed Nov 16, 2020).

20. Kumari A, Yadav K, Yadav C. Biodegradable polymeric nanoparticles based drug delivery systems. Colloids and surfaces B: biointerfaces. 2010. 75(1), 1-18. https://www.sciencedirect.com/science/article/abs/pii/S0927776509004111. (accessed Nov 16, 2020).

21. Carrasquillo G, Stanley M, Aponte-Carro C, De Jésus P, Costantino R, Bosques J, Griebenow K. Non-aqueous encapsulation of excipient-stabilized spray-freeze dried BSA into poly (lactide-co-glycolide) microspheres results in release of native protein. Journal of Controlled $\quad$ Release. $2001 . \quad 76(3), \quad 199-208$. https://www.sciencedirect.com/science/article/abs/pii/S0168365901004308. (accessed Nov 16, 2020).

22. Di Toro R, Betti V, Spampinato S. Biocompatibility and integrin-mediated adhesion of human osteoblasts to poly (DL-lactide-co-glycolide) copolymers. European journal of $\begin{array}{llll}\text { pharmaceutical } & \text { sciences. } & 2004 . & \text { 21(2-3), }\end{array}$ https://pubmed.ncbi.nlm.nih.gov/14757487/. (accessed Nov 16, 2020).

23. Torchilin P. Multifunctional nanocarriers. Advanced drug delivery reviews. 2006. 58(14), 15321555. https://pubmed.ncbi.nlm.nih.gov/17092599/. (accessed Nov 16, 2020).

24. Derakhshandeh K, Erfan M, Dadashzadeh S. Encapsulation of 9-nitrocamptothecin, a novel anticancer drug, in biodegradable nanoparticles: factorial design, characterization and release kinetics. European journal of pharmaceutics and biopharmaceutics. 2007. 66(1), 34-41. https://pubmed.ncbi.nlm.nih.gov/17070678/. (accessed Nov 16, 2020).

25. Fonseca C, Simoes S, Gaspar R. Paclitaxel-loaded PLGA nanoparticles: preparation, physicochemical characterization and in vitro anti-tumoral activity. Journal of controlled release. 2002. 83(2), 273-286. https://pubmed.ncbi.nlm.nih.gov/12363453/. (accessed Nov 16, 2020).

26. Avgoustakis K, Beletsi A, Panagi Z, Klepetsanis P, Karydas G, Ithakissios S. PLGA-mPEG nanoparticles of cisplatin: in vitro nanoparticle degradation, in vitro drug release and in vivo drug residence in blood properties. Journal of controlled release. 2002. 79(1-3), 123135. https://pubmed.ncbi.nlm.nih.gov/11853924/. (accessed Nov 16, 2020).

27. Biswas S, Kumari P, Lakhani M, Ghosh B. Recent advances in polymeric micelles for anticancer drug delivery. European Journal of Pharmaceutical Sciences. 2016. 83, 184-202. https://pubmed.ncbi.nlm.nih.gov/26747018/. (accessed Nov 16, 2020).

28. Rowe C, Sheskey P, Quinn M. Handbook of pharmaceutical excipients. Libros DigitalesPharmaceutical Press. 2009. http://repositorio.ub.edu.ar/handle/123456789/5143. (accessed Nov 16, 2020).

29. Zhao L, Du J, Duan Y, Zhang H, Yang C, Cao F, Zhai G. Curcumin loaded mixed micelles composed of Pluronic P123 and F68: preparation, optimization and in vitro characterization. Colloids and Surfaces B: Biointerfaces. 2012. 97, 101-108. https://pubmed.ncbi.nlm.nih.gov/22609589/. (accessed Nov 16, 2020). 
30. Park H, Park W, Na K. Doxorubicin loaded singlet-oxygen producible polymeric micelle based on chlorine e6 conjugated pluronic F127 for overcoming drug resistance in cancer. Biomaterials. 2014. 35(27), 7963-7969. https://pubmed.ncbi.nlm.nih.gov/24934645/. (accessed Nov 16, 2020).

31. Ren J, Fang Z, Yao L, Dahmani Z, Yin L, Zhou J, Yao J. A micelle-like structure of poloxamermethotrexate conjugates as nanocarrier for methotrexate delivery. International Journal of Pharmaceutics. 2015. 487(1-2), 177-186. https://pubmed.ncbi.nlm.nih.gov/25865570/. (accessed Nov 16, 2020).

32. Tang X, Liang Y, Feng X, Zhang R, Jin X, Sun L. Co-delivery of docetaxel and Poloxamer 235 by PLGA-TPGS nanoparticles for breast cancer treatment. Materials Science and $\begin{array}{llll}\text { Engineering: } & C & 2015 . & 49,\end{array}$ https://www.sciencedirect.com/science/article/abs/pii/S0928493115000430. (accessed Nov 16, 2020).

33. Kulkarni A, Caporali P, Dolas A. Linear Cyclodextrin Polymer Prodrugs as Novel Therapeutics for Niemann-Pick Type C1 Disorder. Scientific Reports. 2018. 8, 9547. https://doi.org/10.1038/s41598-018-27926-9. (accessed Nov 16, 2020).

34. Arms L, Smith W, Flynn J, Palmer W, Martin A, Woldu A, Hua S. Advantages and limitations of current techniques for analyzing the biodistribution of nanoparticles. Frontiers in pharmacology. 2018.

9 , 802. https://www.frontiersin.org/articles/10.3389/fphar.2018.00802/full. (accessed Dec 11, 2020). 